\title{
A Comparative Study of Single-Ended vs. Differential Six-Port Modulators for Wireless Communications
}

J oakim Östh, Magnus Karlsson, Adriana Serban and Shaofang Gong

The self-archived postprint version of this journal article is available at Linköping University Institutional Repository (DiVA):

http:// urn.kb.se/ resolve?urn=urn:nbn:se:liu:diva-115331

N.B.: When citing this work, cite the original publication.

Östh, J ., Karlsson, M., Serban, A., Gong, S., (2015), A Comparative Study of Single-Ended vs.

Differential Six-Port Modulators for Wireless Communications, IEEE Transactions on Circuits and Systems Part 1, 62(2), 564-570. https:/ / doi.org/ 10.1109/ TCSI.2014.2362310

Original publication available at:

https:// doi.org/ 10.1109/ TCSI.2014.2362310

Copyright: Institute of Electrical and Electronics Engineers (IEEE)

http:// www.ieee.org/ index.html

(C)2015 IEEE. Personal use of this material is permitted. However, permission to reprint/republish this material for advertising or promotional purposes or for creating new collective works for resale or redistribution to servers or lists, or to reuse any copyrighted component of this work in other works must be obtained from the IEEE. 


\title{
A Comparative Study of Single-Ended vs Differential Six-Port Modulators for Wireless Communications
}

\author{
Joakim Östh, Magnus Karlsson, Adriana Serban and Shaofang Gong Member, IEEE
}

\begin{abstract}
If present, nonlinear effects in a six-port modulator cause distortion and degradation of the quality of the modulated output waveform. How nonlinear effects occur and their impact on system performance were derived in a mathematical model. The model shows that non-ideal performance of the passive sixport correlator is the main contributor to nonlinear distortion. Simulations and measurements on two manufactured six-port modulators were used to validate the theory and to give deeper insight on system performance. It is shown that by using a differentially signaled six-port modulator instead of a singleended six-port modulator, better performance is achieved over a wide bandwidth. For an error vector magnitude of less than $10 \%$, the relative bandwidth was measured to $12 \%$ for the single-ended but $30 \%$ for the differentially signaled modulator.
\end{abstract}

Index Terms-six-port correlator, six-port modulator, nonlinearity, modulation order, six-port, differential, single-ended.

\section{INTRODUCTION}

$\mathbf{T}$ HERE have been much interest recently in using sixport correlators in communication devices [1]-[16]. Many studies have been done on demodulators where a six-port correlator is used together with power detectors to generate the baseband in-phase (I) and quadrature-phase (Q) data [2][7], [17]. For modulation, the six-port correlator can instead be used together with variable impedance loads to generate the modulated radio frequency (RF) signal. The variable impedance loads are used to generate different reflection coefficients on the respective ports of the six-port correlator, which in turn modulates an applied carrier signal [5], [8], [9], [18], [19]. The variable load can, for example, be implemented with transistors [10], [11], [18], [20], switches [5], [9] or diodes [12], [13]. As stated by Shannon and Nyquist, the key factors of communication are bandwidth, number of signal levels, and signal-to-noise ratio [15]. The six-port technology has potential in communication systems due to its strength in all the three key factors [13]. However, a real system ought to be affected by various non-idealities, e.g., carrier leakage may occur when using variable loads together with a sixport correlator for modulation [8], [11]. Carrier leakage has a negative impact on both the error vector magnitude (EVM) and channel capacity [21] and introduces dc-offset in the receiver [8], [22]. A technique to suppress the carrier leakage

Manuscript received May 23, 2014; revised Aug. 13, 2014.

Joakim Östh; email: joakim.osth@gmail.com, et al., are with Linköping University, Sweden.

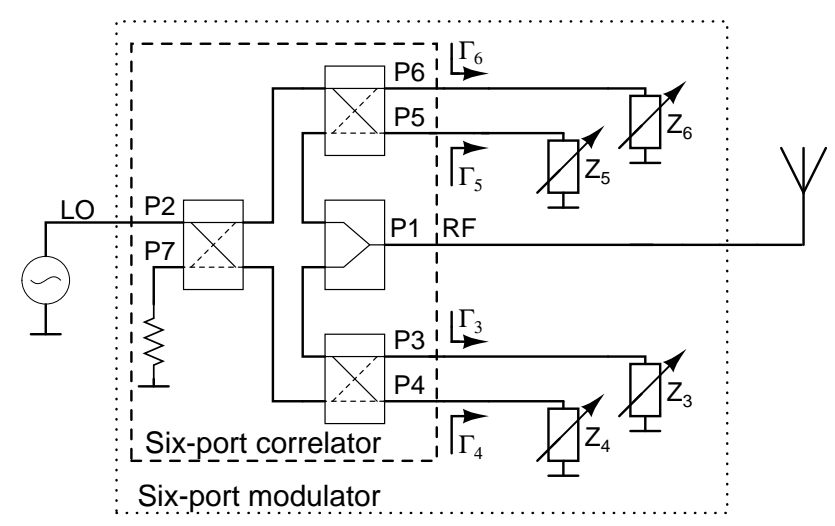

Figure 1. Schematic of a six-port modulator.

was previously presented in [13] that also ought to improve the EVM performance. In a real six-port correlator circuit, there are unwanted effects that may cause carrier leakage, crosstalk between I and Q channels and nonlinear distortion of the modulated RF signal. It has been shown that a strongly coupled differential six-port architecture (broadside-coupled) can reduce some of these issues [23], [24], but no clear conclusion was given. Moreover, the previous work has not addressed if the improvement is owing the strongly broadsidecoupled structure or to the differential signaling itself. The reasons for carrier leakage and nonlinear distortion and their impact on system performance are investigated in this paper. Both a single-ended and a side-by-side differential architecture are investigated, i.e., the differential circuit in this case consists of two single-ended modulators that are differentially fed.

\section{A. Principle of the Six-Port as Modulator}

A conventional six-port correlator [1], [13]-[15] is shown in the dashed-line box in Fig. 1. It consists of one power divider and three $90^{\circ}$ branch-line couplers. If an incident wave $\left(a_{2}\right)$ is applied on port $\mathrm{P} 2$, this wave experiences different phase shifts when it passes through the six-port correlator to each of the four output ports (P3 - P6). The transmitted outgoing waves on ports (P3 - P6), due to the input wave at port P2, travel along the transmission lines and eventually get reflected at the impedance loads $Z_{3}-Z_{6}$. The reflected wave now becomes the input on ports (P3 - P6) and a part of the merged waves is transferred to the output port P1. Due to the internal phase relations in the six-port correlator, and depending on how the impedance loads $\left(Z_{3}-Z_{6}\right)$ are selected, a modulated signal 
containing both I and Q data can then be directly generated, if the impedance loads are controlled by a baseband signal. In an ideal six-port modulator it is shown in [11], [13] that the modulated output wave $b_{1}$ at port P1 is

$$
b_{1}=-\frac{a_{2}}{4}\left[\left(\Gamma_{3}+\Gamma_{4}\right)+j\left(\Gamma_{5}+\Gamma_{6}\right)\right]
$$

where $a_{2}$ is the forward wave at port P2. $\Gamma_{3}, \Gamma_{4}, \Gamma_{5}$ and $\Gamma_{6}$ are the reflection coefficients at ports P3, P4, P5 and P6, i.e., defined looking into the loads. Clearly, $b_{1}$ can obtain any complex modulated value if $\Gamma_{3}$ to $\Gamma_{6}$ are arbitrary set. However, in a real circuit, there will be distortions in the output signal compared to the ideal case. The parameters that are critical for a six-port as a modulator are described in the following sections.

\section{THEORY}

A branch-line coupler is terminated with variable loads $Z_{2}$ and $Z_{3}$ as illustrated in Fig. 2. Assume that an input carrier

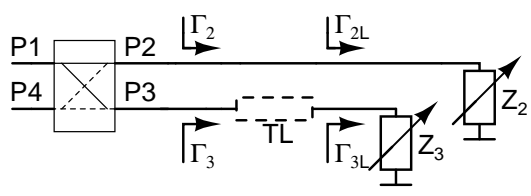

Figure 2. Schematic of a hybrid coupler connected to variable impedance loads.

wave $a_{1}$ is applied on the input port P1 of the coupler, and $a_{4}$ is the incident wave at port 4 . Then the reflection coefficient seen at port $\mathrm{P} 2$ and $\mathrm{P} 3$ are $\Gamma_{2}$ and $\Gamma_{3}$. The output wave $b_{4}$ is present at port P4. From S-parameter analysis:

$$
\begin{gathered}
b_{x}=S_{x 1} a_{1}+S_{x 2} a_{2}+S_{x 3} a_{3}+S_{x 4} a_{4}, \quad \forall x \in\{1,2,3,4\} \\
a_{2}=\Gamma_{2} b_{2} \\
a_{3}=\Gamma_{3} b_{3} \\
b_{x}=S_{x 1} a_{1}+S_{x 2} \Gamma_{2} b_{2}+S_{x 3} \Gamma_{3} b_{3}+S_{x 4} a_{4}, \quad \forall x \in\{1,2,3,4\} \\
b_{4}=S_{41} a_{1}+S_{42} \Gamma_{2} b_{2}+S_{43} \Gamma_{3} b_{3}+S_{44} a_{4} \\
b_{2}=\frac{S_{21} a_{1}+S_{23} \Gamma_{3} b_{3}+S_{24} a_{4}}{1-S_{22} \Gamma_{2}} \\
b_{3}=\frac{S_{31} a_{1}+S_{32} \Gamma_{2} b_{2}+S_{34} a_{4}}{1-S_{33} \Gamma_{3}}
\end{gathered}
$$

solving (7) and (8) for $b_{2}$ and $b_{3}$ and using the results in (6) the total output wave $b_{4}$ can be expressed in terms of the input wave $a_{1}, a_{4}$, and the two reflection coefficients $\Gamma_{2}$ and $\Gamma_{3}$. Due to the symmetry of the coupler:

$$
\begin{aligned}
& S_{12}=S_{21}=S_{34}=S_{43}=S_{D} \\
& S_{13}=S_{31}=S_{24}=S_{42}=S_{C} \\
& S_{11}=S_{22}=S_{33}=S_{44}=S_{R} \\
& S_{14}=S_{41}=S_{23}=S_{32}=S_{I}
\end{aligned}
$$

where the following S-parameter notations are used: $S_{D}$ the direct path, $S_{C}$ the coupled path, $S_{R}$ the input reflection and $S_{I}$ the isolation of the coupler. Furthermore, it will be assumed that there is no input wave at port P4, i.e., $a_{4}=0$.

\section{A. Case 1 - Output Signal - Carrier Leakage Suppressed}

When carrier leakage is suppressed, TL in Fig. 2 is chosen to be a quarter wavelength, e.g., as in [13], $\Gamma_{2}, \Gamma_{3}$ can then be defined as

$$
\begin{gathered}
\Gamma_{2}=\Gamma_{2 L}=\Gamma_{C M}+\Delta \Gamma \\
\Gamma_{3}=-\Gamma_{3 L}=-\Gamma_{C M}+\Delta \Gamma
\end{gathered}
$$

where $\Gamma_{C M}$ is a static or common mode part and $\Delta \Gamma$ a time varying part that allows for modulation. The termination loads $Z_{2}$ and $Z_{3}$ are controlled to result into the desired symbol constellation. Using (6) - (14) and $a_{4}=0$ results in:

$$
b_{4}=\left[\alpha_{0}+\alpha_{1} \Delta \Gamma+\alpha_{2}\left(\Delta \Gamma^{2}-\Gamma_{C M}^{2}\right)\right] a_{1}
$$

where

$$
\begin{aligned}
& \alpha_{0}=\frac{S_{I}}{1-2 S_{R} \Delta \Gamma-\left(S_{I}^{2}-S_{R}^{2}\right)\left(\Delta \Gamma^{2}-\Gamma_{C M}^{2}\right)} \\
& \alpha_{1}=\frac{2 S_{C} S_{D}-2 S_{I} S_{R}}{1-2 S_{R} \Delta \Gamma-\left(S_{I}^{2}-S_{R}^{2}\right)\left(\Delta \Gamma^{2}-\Gamma_{C M}^{2}\right)} \\
& \alpha_{2}=\frac{\left(S_{C}^{2}+S_{D}^{2}+S_{R}^{2}-S_{I}^{2}\right) S_{I}-2 S_{C} S_{D} S_{R}}{1-2 S_{R} \Delta \Gamma-\left(S_{I}^{2}-S_{R}^{2}\right)\left(\Delta \Gamma^{2}-\Gamma_{C M}^{2}\right)}
\end{aligned}
$$

For an ideal coupler, $S_{I}=S_{R}=0, S_{D}=-j / \sqrt{2}, S_{C}=$ $-1 / \sqrt{2}$ and hence $\alpha_{0}=0, \alpha_{1}=j, \alpha_{2}=0$. The ideal output wave $b_{4, i d e a l \_1}$ is

$$
b_{4, i d e a l_{-} 1}=j \triangle \Gamma a_{1}
$$

\section{B. Case 2 - Output Signal - Carrier Leakage Present}

When TL in Fig. 2 is zero and the same reflection coefficient at port $\mathrm{P} 2$ and $\mathrm{P} 3$ are used, i.e., $Z_{2}$ and $Z_{3}$ are the same,

$$
\Gamma_{2}=\Gamma_{2 L}=\Gamma_{3}=\Gamma_{3 L}=\Gamma=\Gamma_{C M}+\Delta \Gamma
$$

using (6) - (12), (20) and $a_{4}=0$ and solving for $b_{4}$ results in

$$
b_{4}=\left(\alpha_{0}+\alpha_{1} \Gamma+\alpha_{2} \Gamma^{2}\right) a_{1}
$$

where

$$
\begin{gathered}
\alpha_{0}=\frac{S_{I}}{1-2 S_{R} \Gamma-\left(S_{I}^{2}-S_{R}^{2}\right) \Gamma^{2}} \\
\alpha_{1}=\frac{2 S_{C} S_{D}-2 S_{I} S_{R}}{1-2 S_{R} \Gamma-\left(S_{I}^{2}-S_{R}^{2}\right) \Gamma^{2}} \\
\alpha_{2}=\frac{\left(S_{C}^{2}+S_{D}^{2}+S_{R}^{2}-S_{I}^{2}\right) S_{I}-2 S_{C} S_{D} S_{R}}{1-2 S_{R} \Gamma-\left(S_{I}^{2}-S_{R}^{2}\right) \Gamma^{2}}
\end{gathered}
$$

For an ideal coupler, the ideal output wave $b_{4, i d e a l \_} 2$ in this case is

$$
b_{4, i d e a l \_2}=j \Gamma a_{1}=j\left(\Gamma_{C M}+\Delta \Gamma\right) a_{1}
$$

In a non-ideal coupler the output $b_{4}$ is nonlinearly dependent on $\Delta \Gamma$ and $\Gamma_{C M}$ according to (15) - (18) for Case 1, and according to (21) - (24) for Case 2. $\alpha_{0}, \alpha_{1}$ and $\alpha_{2}$ are all nonlinearly dependent on $\Gamma$. However, assuming $\left|S_{I}\right|<<1$ and $\left|S_{R}\right|<<1$ the denominator in (16) - (18) can be approximated to be equal to 1 . With that assumption, it is seen from (15) and (21) that in both cases, the carrier leakage 
to $b_{4}$ appears due to $\alpha_{0} \neq 0 \approx S_{I}$. The term $\alpha_{1}$ relates linearly the output signal $b_{4}$ to $\triangle \Gamma$ for Case 1 and to $\Gamma=\Gamma_{C M}+\triangle \Gamma$ for Case 2. Note that for Case 2, also $\alpha_{1}$ adds carrier leakage to the output $b_{4} . \alpha_{2}$ contributes in both Case 1 and Case 2, with nonlinear distortion through $\alpha_{2} \neq 0$ as $b_{4}$ depends on the second-order reflection coefficient terms, as seen from (15) and (21).

In the following sections $\alpha_{0}^{\prime}, \alpha_{1}^{\prime}$ and $\alpha_{2}^{\prime}$ are used to denote ideal case when the denominator of $\alpha_{0}, \alpha_{1}$ and $\alpha_{2}$ are 1, i.e., independent on the value of $\Delta \Gamma$ and $\Gamma_{C M}$ (Case 1) or $\Gamma$ (Case 2 ). To avoid unnecessary indexing, $b=b_{4}$ and $a=a_{1}$.

\section{ANALYSis OF Single-ENDED VS DifFERENTIAL SIX-PORT MODULATORS}

The differential six-port circuit used in this case is defined to be two identical single-ended six-port modulators with the two local oscillator (LO) signals $a_{p}$ and $a_{n}$ differential, i.e., related by $a_{n}=-a_{p}$. The two outputs, $b_{p}$ and $b_{n}$, are added in phase. In addition, the time varying part of the reflection coefficient, $\Delta \Gamma$, is also differential:

$$
\begin{aligned}
& \Gamma_{p}=\Gamma_{C M}+\Delta \Gamma \\
& \Gamma_{n}=\Gamma_{C M}-\Delta \Gamma
\end{aligned}
$$

\section{A. Carrier Leakage and Nonlinear Distortion}

The different mechanisms that generate carrier leakage and nonlinear distortion are considered and compared for the single-ended and differential six-port modulators.

1) Single-Ended Circuit: One way to limit the impact of carrier leakage from a static part in the reflection coefficient $\Gamma$ was presented in [13] and may be used for both single-ended and differential circuits. The technique for carrier leakage suppression is utilized, i.e., Case 1 when TL in Fig. 2 is a quarter of a wavelength,

$$
b=\left[\alpha_{0}+\alpha_{1} \Delta \Gamma+\alpha_{2}\left(\Delta \Gamma^{2}-\Gamma_{C M}^{2}\right)\right] a
$$

It is also common to use the same reflection coefficient at port P2 and P3, which corresponds to Case 2. In this case the output signal is given by (21). Moreover, by assuming that the reflection coefficient $\Gamma$ has both a static common mode component $\Gamma_{C M}$ and a time varying component $\Delta \Gamma$, i.e.,

$$
\begin{gathered}
\Gamma=\Gamma_{C M}+\Delta \Gamma \\
b=\left[\alpha_{0}+\alpha_{1}\left(\Gamma_{C M}+\Delta \Gamma\right)+\alpha_{2}\left(\Gamma_{C M}+\Delta \Gamma\right)^{2}\right] a
\end{gathered}
$$

In both cases, for a non-ideal coupler $\alpha_{0} \neq 0$ and $\alpha_{2} \neq 0$ so there is a carrier leakage, independent of $\Gamma_{C M}$ and $\Delta \Gamma$. Moreover, because $\alpha_{2} \neq 0$ there is a nonlinear distortion added to the output signal. If the same reflection coefficient is used on port P2 and P3, it is seen from (30) that there is a carrier leakage if $\Gamma_{C M} \neq 0$ even if an ideal coupler is used, i.e., $\alpha_{0}=\alpha_{2}=0$. This problem is not present when the technique for carrier leakage suppression is used, as evident from (28). A serious problem is the nonlinear distortion owing to $\alpha_{2} \neq 0$ as this limits the bandwidth where a specified EVM can be obtained.
2) Differential Circuit: One way to suppress the carrier leakage from $\alpha_{0}$ is to use a differential six-port circuit, i.e., use two six-port circuits where the two LO signals $a_{p}$ and $a_{n}$ are related by $a_{n}=-a_{p}$ and the two outputs, $b_{p}$ and $b_{n}$, are added in phase. For Case 1 by using (15):

$$
\begin{gathered}
b_{p}=\left[\alpha_{0 p}+\alpha_{1 p} \Delta \Gamma_{p}+\alpha_{2 p}\left(\Delta \Gamma_{p}^{2}-\Gamma_{C M}^{2}\right)\right] a_{p} \\
b_{n}=-\left[\alpha_{0 n}+\alpha_{1 n} \Delta \Gamma_{n}+\alpha_{2 n}\left(\Delta \Gamma_{n}^{2}-\Gamma_{C M}^{2}\right)\right] a_{p}
\end{gathered}
$$

after summing the total output signal is

$$
\begin{aligned}
b=b_{p}+b_{n}= & \alpha_{0 p}-\alpha_{0 n}+\alpha_{1 p} \Delta \Gamma_{p}-\alpha_{1 n} \Delta \Gamma_{n}+ \\
& \alpha_{2 p} \Delta \Gamma_{p}^{2}-\alpha_{2 n} \Delta \Gamma_{n}^{2}-\left(\alpha_{2 p}-\alpha_{2 n}\right) \Gamma_{C M}^{2}
\end{aligned}
$$

For an ideal coupler $\alpha_{m p}=\alpha_{m n}=\alpha_{m}^{\prime}, \forall m \in\{0,1,2\}$, in this case

$$
b_{\text {ideal }}=b_{p}+b_{n}=\alpha_{1}^{\prime}\left(\Delta \Gamma_{p}-\Delta \Gamma_{n}\right)+\alpha_{2}^{\prime}\left(\Delta \Gamma_{p}^{2}-\Delta \Gamma_{n}^{2}\right)
$$

the carrier leakage from $\alpha_{0}^{\prime}$ is hence suppressed. To get a modulated output signal, select $\Delta \Gamma_{n}=\beta \Delta \Gamma_{p}$ where $\beta \neq 1$ and to suppress the nonlinear distortion select $\beta=-1$, in this case

$$
b_{\text {ideal }}=2 \alpha_{1}^{\prime} \Delta \Gamma_{p}
$$

carrier leakage and nonlinear distortion are hence suppressed when a differential six-port circuit is used. The exact output signal is given by (33), but close to the design frequency $\alpha_{m p} \approx \alpha_{m n}, \forall m \in\{0,1,2\}$ and (35) may be used as a first approximation.

Similarly, for Case 2 when the same reflection coefficients are used on port P2 and P3, by using (21):

$$
\begin{gathered}
b_{p}=\left(\alpha_{0 p}+\alpha_{1 p} \Gamma_{p}+\alpha_{2 p} \Gamma_{p}^{2}\right) a_{p} \\
b_{n}=-\left(\alpha_{0 n}+\alpha_{1 n} \Gamma_{n}+\alpha_{2 n} \Gamma_{n}^{2}\right) a_{p}
\end{gathered}
$$

after summing the total output signal $b=b_{p}+b_{n}$ is

$$
b=\left(\alpha_{0 p}-\alpha_{0 n}+\alpha_{1 p} \Gamma_{p}-\alpha_{1 n} \Gamma_{n}+\alpha_{2 p} \Gamma_{p}^{2}-\alpha_{2 n} \Gamma_{n}^{2}\right) a_{p}
$$

For an ideal coupler, $\alpha_{m p}=\alpha_{m n}=\alpha_{m}^{\prime}, \forall m \in\{0,1,2\}$, in this case the total output signal is

$$
b_{\text {ideal }}=b_{p}+b_{n}=\left[\alpha_{1}^{\prime}\left(\Gamma_{p}-\Gamma_{n}\right)+\alpha_{2}^{\prime}\left(\Gamma_{p}^{2}-\Gamma_{n}^{2}\right)\right] a_{p}
$$

To get a modulated output signal and to suppress nonlinear distortion, $\Gamma_{p}$ and $\Gamma_{n}$ are selected according to (26) and (27), this results in

$$
b_{\text {ideal }}=\left(2 \alpha_{1}^{\prime} \Delta \Gamma+4 \alpha_{2}^{\prime} \Gamma_{C M} \Delta \Gamma\right) a_{p}
$$

Close to the design frequency $\alpha_{m p} \approx \alpha_{m n}, \forall m \in\{0,1,2\}$ and (40) may be used as an approximation. But the exact output is given by (38).

In both cases, the carrier leakage and nonlinear distortion are suppressed when a differential six-port circuit is used. There is no need to use any additional carrier leakage suppression technique when a differential six-port circuit is used, and lower circuit complexity can thus be obtained by using the same reflection coefficients (and hence loads) at ports P3 and P4. Moreover, the differential six-port circuit results 
in a broader bandwidth than that using a carrier leakage suppression technique since the phase shifter (TL) shown in Fig. 2 is not needed.

\section{B. $E V M$}

The total transmitted signal $b_{T X}$ is generated from the independent I and Q channel, the LO signal $a_{L O}$

$$
b_{T X}=k\left(b_{I}+j b_{Q}\right) a_{L O}
$$

and the error

$$
\begin{aligned}
e_{T X} & =b_{T X}-b_{T X}^{\prime}=k\left[b_{I}-b_{I}^{\prime}+j\left(b_{Q}-b_{Q}^{\prime}\right)\right] a_{L O} \\
& =k\left(e_{I}+j e_{Q}\right) a_{L O} \\
\left|e_{T X}\right|^{2} & =|k|^{2}\left(\left|e_{I}\right|^{2}+\left|e_{Q}\right|^{2}+2\left|e_{I}\right|\left|e_{Q}\right| \cos \phi\right)\left|a_{L O}\right|^{2}
\end{aligned}
$$

where

$$
\phi=\angle e_{I}-\angle e_{Q}
$$

$b_{T X}, b_{I}, b_{Q}$ are the actual signals, $b_{T X}^{\prime}, b_{I}^{\prime}, b_{Q}^{\prime}$ the ideal signals, and $k$ a scaling factor. Because I and Q channels are assumed to have identical circuits for generation of the required reflection coefficients, only one arbitrary selected path is considered. For EVM calculation the transmitted reference signal is assumed to be of the form

$$
S_{T X, r e f}=\Delta \Gamma
$$

The actually transmitted signal, for the case when the carrier leakage suppression technique is used of the form

$$
S_{T X}=A e^{j \phi}\left[\alpha_{0}+\alpha_{1} \Delta \Gamma+\alpha_{2}\left(\Delta \Gamma^{2}-\Gamma_{C M}^{2}\right)\right]
$$

where $A$ is an amplitude scaling and $\phi$ a phase shift that occurs in the circuit. The error

$$
\begin{aligned}
S_{\text {error }}= & S_{T X}-S_{T X, \text { ref }} \\
= & A e^{j \phi}\left[\alpha_{0}+\alpha_{2}\left(\Delta \Gamma^{2}-\Gamma_{C M}^{2}\right)\right]+ \\
& A e^{j \phi}\left(\alpha_{1}-\frac{1}{A e^{j \phi}}\right) \Delta \Gamma
\end{aligned}
$$

can only be zero when $\alpha_{0}=\alpha_{2}=0$ and $A e^{j \phi}=1 / \alpha_{1}$. Because the denominator of $\alpha_{0}, \alpha_{1}$ and $\alpha_{2}$ are dependent on the reflection coefficient, as seen from (16) - (18) and (22) (24), the condition $A e^{j \phi}=1 / \alpha_{1}$ cannot in general be fulfilled, which results in a small error. However, the variation in the denominator of $\alpha_{0}, \alpha_{1}$ and $\alpha_{2}$ with the reflection coefficient is assumed to be small around the design frequency, in which case the condition $A e^{j \phi} \approx 1 / \alpha_{1}$ can be achieved. Deriving closed form formulas for the EVM would be quite extensive, hence some approximations will be used in the following sub sections.

1) Single-Ended Circuit: First the contribution to the EVM error for Case 1, i.e., when the technique for carrier leakage suppression is utilized, is estimated from (28):

$$
\begin{gathered}
y=b / a=\alpha_{0}+\alpha_{1} \Delta \Gamma+\alpha_{2}\left(\Delta \Gamma^{2}-\Gamma_{C M}^{2}\right) \\
y_{i}=\left.y\right|_{\alpha_{0}=\alpha_{2}=0}=\alpha_{1}^{\prime} \Delta \Gamma
\end{gathered}
$$

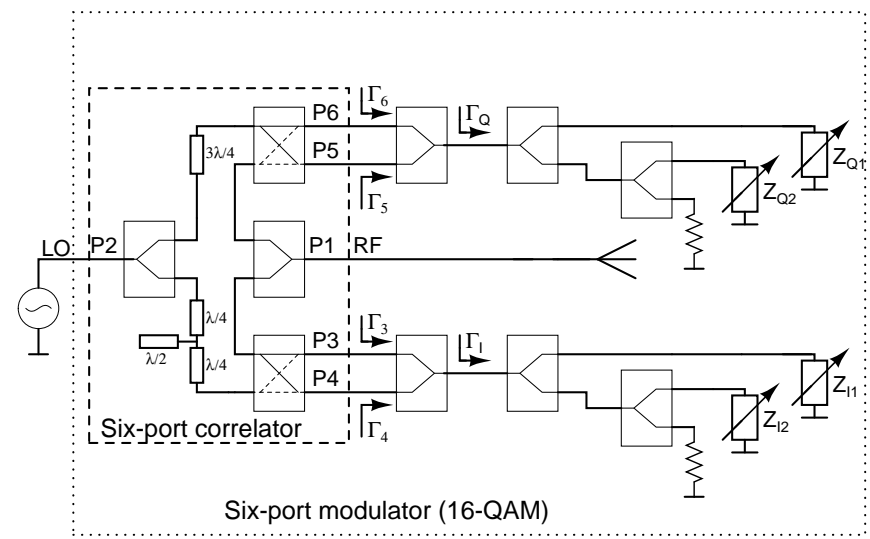

Figure 3. Schematic of implemented six-port modulator.

$$
\begin{aligned}
y_{e} & =y-y_{i}=\alpha_{0}+\left(\alpha_{1}-\alpha_{1}^{\prime}\right) \Delta \Gamma+\alpha_{2}\left(\Delta \Gamma^{2}-\Gamma_{C M}^{2}\right) \\
& \approx \alpha_{0}+\alpha_{2}\left(\Delta \Gamma^{2}-\Gamma_{C M}^{2}\right)
\end{aligned}
$$

where $y$ is the actual output signal, $y_{i}$ the ideal output signal, and $y_{e}$ the error. As $\alpha_{1}$ is very close to $\alpha_{1}^{\prime}$ within the bandwidth around the center frequency, the difference $\alpha_{1}-\alpha_{1}^{\prime} \rightarrow 0$ in (50). Hence, for the single-ended case the error $y_{e}$ in (50) shows an nonlinear dependence on $\Delta \Gamma$ and $\Delta_{C M}$.

$$
\begin{aligned}
\left|y_{e}\right|^{2} \approx & \left|\alpha_{0}\right|^{2}+\left|\alpha_{2}\right|^{2}\left|\Delta \Gamma^{2}-\Gamma_{C M}^{2}\right|^{2}+ \\
& 2\left|\alpha_{0}\right|\left|\alpha_{2}\right|\left|\Delta \Gamma^{2}-\Gamma_{C M}^{2}\right| \cos \phi_{S E}
\end{aligned}
$$

where

$$
\phi_{S E}=\angle \alpha_{0}-\angle \alpha_{2}-\angle\left(\Delta \Gamma^{2}-\Gamma_{C M}^{2}\right)
$$

As seen, the non-ideal performance of the coupler introduces distortions to the modulated signal and hence degrades the EVM. For high efficiency, i.e., to maximize the output power it is required that $\left|\Gamma_{C M}\right|<<|\Delta \Gamma|$ and in the ideal case $\left|\Gamma_{C M}\right| \rightarrow 0$ so the error is approximately

$$
\left|y_{e}\right|^{2} \approx\left|\alpha_{0}\right|^{2}+\left|\alpha_{2}\right|^{2}\left|\Delta \Gamma^{2}\right|^{2}+2\left|\alpha_{0}\right|\left|\alpha_{2}\right|\left|\Delta \Gamma^{2}\right| \cos \phi_{S E}
$$

and therefore there is a serious degradation of the modulation performance in a single-ended system. If the same reflection coefficients are used on both ports, corresponding to Case 2, a similar expression can be derived. In both cases there is a static error from $\alpha_{0}$ and a nonlinear error from the part $\alpha_{2}$.

2) Differential Circuit: The EVM when a differential sixport is used is estimated from (40), which corresponds to Case 2.

$$
\begin{gathered}
y=b / a=2 \alpha_{1} \Delta \Gamma+4 \alpha_{2} \Gamma_{C M} \Delta \Gamma \\
y_{i}=\left.y\right|_{\alpha_{0}=\alpha_{2}=0}=2 \alpha_{1}^{\prime} \Delta \Gamma \\
y_{e}=y-y_{i}=2\left(\alpha_{1}-\alpha_{1}^{\prime}\right) \Delta \Gamma+4 \alpha_{2} \Gamma_{C M} \Delta \Gamma \\
\approx 4 \alpha_{2} \Gamma_{C M} \Delta \Gamma
\end{gathered}
$$

where it was assumed that $\alpha_{1} \approx \alpha_{1}^{\prime}$ in the neighborhood of the design frequency. Observe that, in contrast to the single-ended case, the error in (56) is only linearly dependent on $\Delta \Gamma$.

$$
\left|y_{e}\right|^{2} \approx 16\left|\alpha_{2}\right|^{2}\left|\Gamma_{C M}\right|^{2}|\Delta \Gamma|^{2}
$$




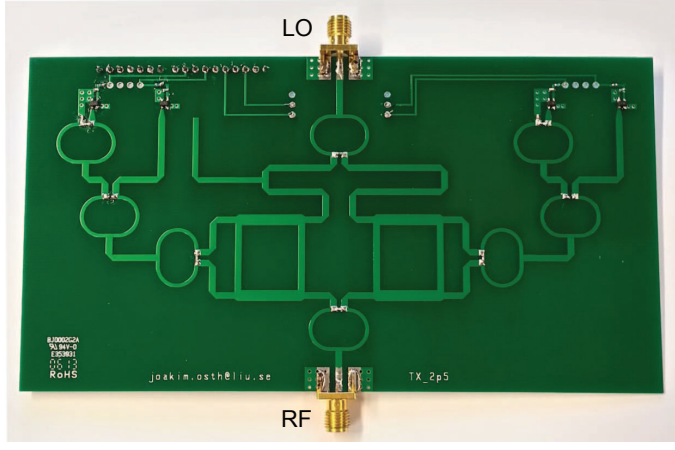

(a)

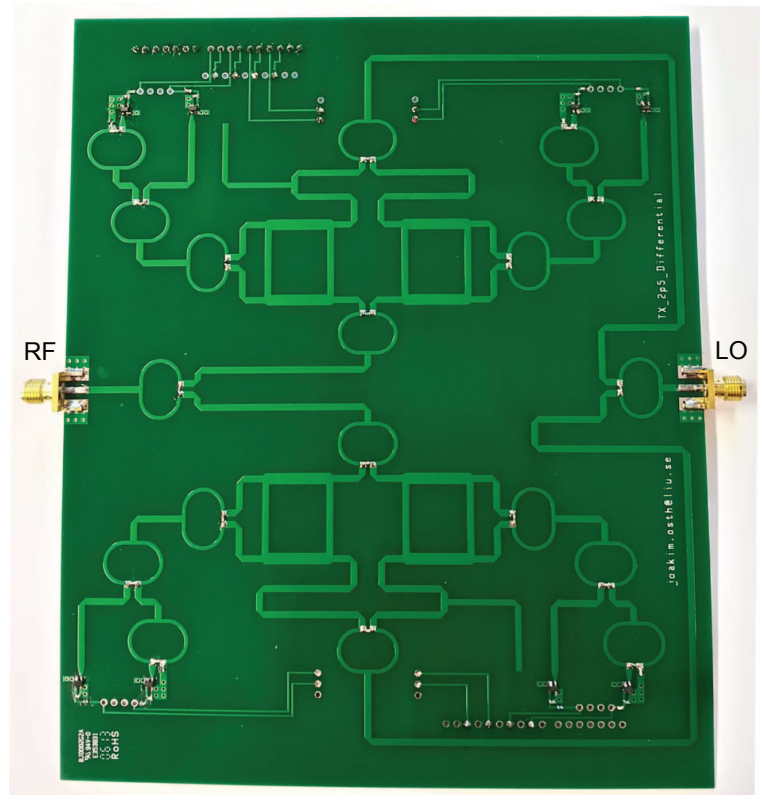

(b)

Figure 4. Photo of the modulator prototypes: (a) single-ended, and (b) differential.

For the case when the technique for carrier leakage suppression is used, i.e., Case 1, the corresponding error is found by setting $\Gamma_{C M}=0$ in (57). For high efficiency, i.e., to maximize the output power it is required that $\left|\Gamma_{C M}\right|<<|\Delta \Gamma|$ and in the ideal case $\left|\Gamma_{C M}\right| \rightarrow 0$ so the (approximate) error in (57) approaches zero.

\section{PRototype Design}

The bandwidth with an acceptable amplitude and phase imbalance and then an acceptable EVM is quite limited in a branch-line coupler. Therefore the coupler responsible for IQ separation is implemented by using a Wilkinson power divider together with a broadband phase shifting network [16], [25]. As seen in Fig. 3, the phase shifting network consists of a $3 \lambda / 4$ transmission line (TL) in one path and a $\lambda / 2$ TL loaded in the center with an open circuit $\lambda / 2 \mathrm{TL}$ in the other path. This allows for a phase shift of about $90^{\circ}$ over a wider bandwidth compared to a branch-line coupler and, hence, increased EVM performance vs frequency.
The modulator is designed for 16-QAM and therefore four different reflection coefficients are required on the I and $Q$ channels. This is accomplished by using a network of cascaded power dividers and two impedance loads. This is shown in Fig. 3 to the right of $\Gamma_{I}$ and $\Gamma_{Q}$. The impedance loads $\left(Z_{I 1}-Z_{I 2}\right.$ and $\left.Z_{Q 1}-Z_{Q 2}\right)$ are implemented by using a FET, ATF58143 from Avago technologies Inc. Each FET operates in a cold-FET configuration and can attain two different (i.e., binary) states, ideally open or short, to maximize the efficiency. It can be shown [19] from S-parameter analysis:

$$
\Gamma_{I}=\frac{2 \Gamma_{I 1}+\Gamma_{I 2}}{4}
$$

and for ideal open and short conditions $4 \Gamma_{I} \in\{-3,-1,1,3\}$, i.e., exactly what is required for 16-QAM modulation. The same holds for the $\mathrm{Q}$ channel. Moreover, because $\Gamma_{3}=\Gamma_{4}$ and $\Gamma_{5}=\Gamma_{6}$, a power divider is connected between the point where $\Gamma_{I}$ and $\Gamma_{Q}$ are present and to the corresponding hybrid coupler in the six-port correlator as shown in Fig. 3. This reduces the number of required impedance loads (FETs) by a factor of two. The drawback is that the magnitude of the reflection coefficient is also reduced by a factor of two. The single-ended and differential six-port modulators are implemented in the exact same way. Differential LO signal is generated by using a Wilkinson power divider and an additional delay of $\lambda / 2$, i.e., $180^{\circ}$ on one path. The prototypes are designed on $0.8 \mathrm{~mm}$ thick FR4 substrate and designed to operate around $f_{0}=2.5 \mathrm{GHz}$. A photo of both the single ended and differential six-port modulator prototypes can be seen in Fig. 4.

\section{RESUlts}

The performance of a single-ended and differential six-port modulator are compared. Both EVM and constellation are investigated. The EVM measured results are derived from $\mathrm{S}$ parameter measurements to be consistent with the presented theoretical analysis performed at S-parameter level. Numerically the exact EVM can be calculated. The EVM [16], [26] is calculated by minimizing the function

$$
E V M=\sqrt{\frac{\sum_{n=0}^{M-1}\left|\frac{R(n)-C_{0}}{C_{1}}-T(n)\right|^{2}}{\sum_{n=0}^{M-1}|T(n)|^{2}}}
$$

where the complex constants $C_{0}$ is an origin offset and $C_{1}$ is used for amplitude scaling and phase rotation. $T(n)$ is the ideal transmitted signal and $R(n)$ the received signal. Observe that the origin offset, i.e., carrier leakage is removed in our EVM calculation.

\section{A. Simulations}

Advanced Design System (ADS) from Agilent Technologies, Inc., was used for simulation of the complete six-port transmitters. The transmitted and received waveforms were exported to Matlab where (59) was used to calculate EVM.

To investigate the performance, a transmission line model of the branch-line hybrid couplers connected to impedance loads is used. The power divider used for I and Q separation 
is ideal and hence will not contribute to the error. The EVM error is only from the non-ideal hybrid coupler and, in the differentially signaled case, from the required $180^{\circ}$ phase shift. Both an ideal phase shift and a phase shift from a $\lambda / 2$ transmission line are compared. The set of used reflection coefficients is ideal and real-valued.

1) EVM: The simulated EVM performance is shown in Fig. 5(a). It can be observed that in the single-ended case a low EVM can only be obtained in a narrow region around the center (design) frequency $f_{0}$. Compared to the single-ended case, a wider bandwidth for a given EVM is obtained with the differential circuit, using either an ideal phase splitter or a $\lambda / 2$ transmission line.

2) Constellation: The constellation at $f=0.8 f_{0}$ for the single-ended case is shown in Fig. 6(a) and for the differential case, when the phase shift is implemented by a $\lambda / 2$ transmission line, in Fig. 7(a). The nonlinear distortion in the single-ended case, predicted by (30) and (50), is clearly seen in Fig. 6(a). A differential circuit results in less nonlinear distortion, see Fig. 7(a).

\section{B. Measurements}

The modulator is characterized by measuring the Sparameters for all possible combinations of the reflection coefficients $\Gamma_{I}$ and $\Gamma_{Q}$. Hence, our prototype modulator for 16-QAM results in a set of 16 different S-parameters. Each set covers a frequency range from 1 to $4 \mathrm{GHz}$. The single-ended case results in one set and the differential case in another set, from which the EVM and constellations are found. The LO power used for measurements was $-13 \mathrm{dBm}$.

1) EVM: The measured EVM performance is found from the measured S-parameters and by using (59) and shown in Fig. 5(b). For the single-ended case, the EVM is less than $10 \%$ from 2.3 to $2.6 \mathrm{GHz}$, i.e., a relative bandwidth of $12 \%$. For the differential circuit, the corresponding range is from 2.0 to $2.7 \mathrm{GHz}$, i.e., a relative bandwidth of $30 \%$.

2) Constellation: The constellation at $f=0.8 f_{0}(2 \mathrm{GHz})$ for the single-ended case is shown in Fig. 6(b) and for the differential case, when the phase shift is implemented by a $\lambda / 2$ transmission line, shown in Fig. 7(b). As predicted, a significant nonlinearity distortion is clearly seen in the singleended case when there is a deviation from the design frequency $f_{0}=2.5 \mathrm{GHz}$. The differential circuit results in less nonlinear distortion, see Fig. 7.

3) Measurement vs Simulation: A good constellation agreement (Fig. 6(a) vs Fig. 6(b) and Fig. 7(a) vs Fig. 7(b)) between simulation and measurement is observed but some clarification is given below. Regarding the EVM results it is seen (Fig. 5(a) vs Fig. 5(b)) that the real behavior of the single-ended in comparison with the differential architecture agrees well with our theoretical analysis and simulation. However, outside the frequency-band of interest the difference is larger due to the imperfections in the real circuit which are not modeled in the simulations. A contribution is due to the fact that the circuit was modeled as separate parts, i.e., using a transmission line model as described before in Section 5.1. Close to the central design frequency the behavior of the circuit is close to ideal, i.e., well matched and all electrical lengths are close to ideal. Further away from the central frequency electrical lengths deviate more from ideal (optimal) electrical lengths. Hence, a larger impact of coupling and crosstalk between circuit parts are present far from the central frequency. For, instance the degrading spike in measured EVM that can be seen in Fig. 5(b) is owing to that fact. Moreover, also the Wilkinson power dividers/combiners add to an increased deviation between simulation and measurement when the frequency is far enough from the design frequency. Hence, far from the design frequency the split is no longer an equal 3-dB split. However, when the simulation vs measurement deviation starts to be noticeable the EVM is already too high to be really useful. Regarding the constellation diagram results it should be noted for comparison the distance to the ideal point is what should be judged, i.e., no phase calibration of the respective symbols with respect to their ideal position is included. Anyway, it is of negligible importance since it is the spread in magnitude from the ideal position that sets the performance boundary.

\section{DISCUSSION}

In Case II in Section II-B, the pair P2, P3 (Fig. 2) was assumed to be equal in the derivation. This means that P2 should be equal to P3, and P4 to P5 in the complete six-port modulator, see Fig. 3. This assumption is done due to a) In the prototypes this was realized with broadband Wilkinson power dividers. Therefore the signal at $\mathrm{P} 3$ will be close to $\mathrm{P} 4$, and $\mathrm{P} 5$ close to P6 within the frequency band of interest, and b) It is known that in general the couplers limit the bandwidth more than the Wilkinson(s) in a six-port modulator/demodulator. Techniques such as stub compensated hybrid couplers have previously been proposed to overcome this [11]. Moreover, also the Wilkinson can be replaced with even more broadband implementations, e.g., multi-stage or tapered (transmission line) implementations [27]. However, improvements of this kind are of little interest in this work since it ought to improve both the single-ended and the differentially signaled solution similarly.

It is obvious from Fig. 4 that the differentially signaled solution has a physical size roughly two times the single-ended solution. However, since the two sides of the differentially signaled six-port circuits do not require any particular electromagnetic coupling between them, they can be placed rather freely in different metal layers in a multi-layer printed circuit board. Hence, in a real application the size difference would not be a crucial point.

Over all it is seen that a differentially signaled six-port modulator has better performance than the single-ended equivalent. Previously, strongly broadside-coupled six-port modulators/demodulators have been studied in [23], [24], a similar improvement of differential architecture over single-ended was observed in those works. Hence, it is likely that the performance improvement in both studies is owing mainly to the differential signaling rather than the physical structure that is used. 


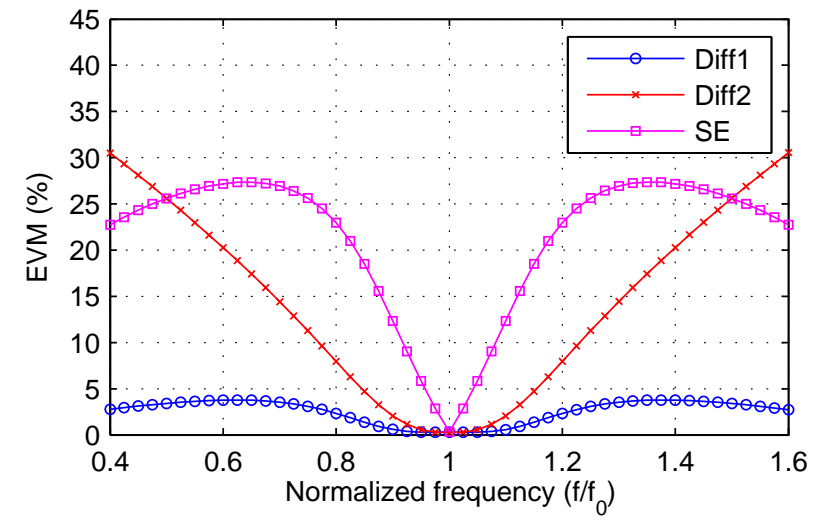

(a)

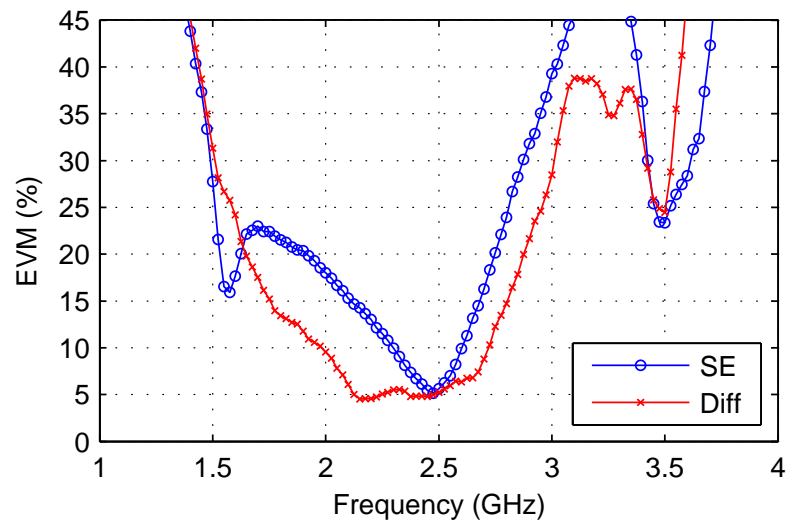

(b)

Figure 5. EVM: (a) simulated EVM vs normalized frequency. Diff1 - ideal phase shift, Diff 2 - phase shift from a $\lambda / 2$ transmission line, and SE - single-ended circuit, and (b) Measured EVM vs frequency for single-ended modulator (SE) and differential modulator (Diff).

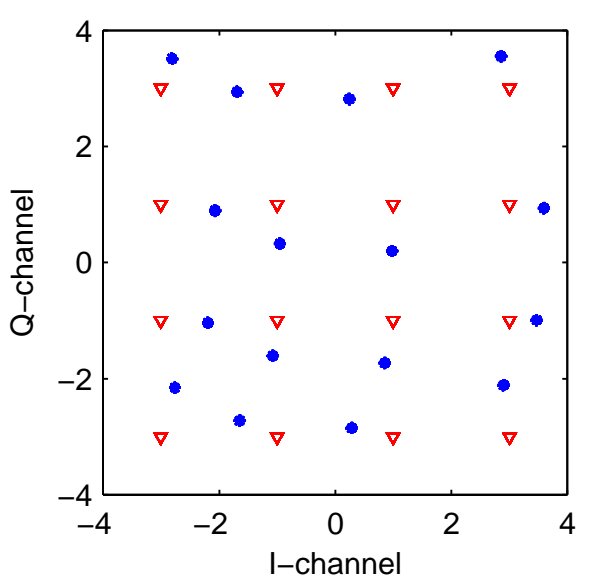

(a)

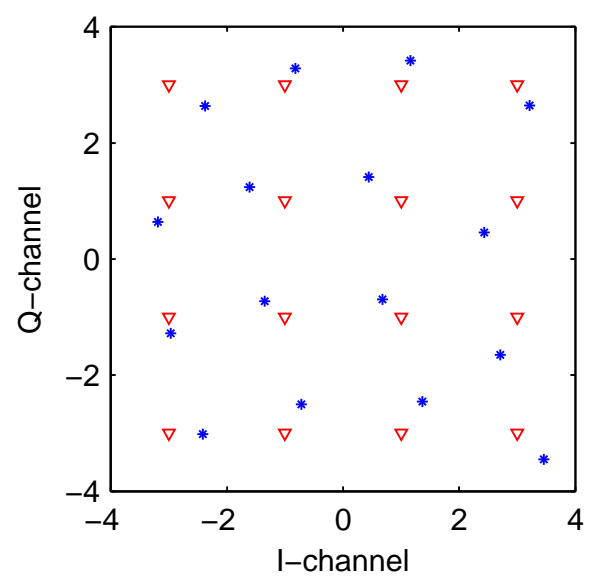

(b)

Figure 6. Constellation at $f=0.8 f_{0}$ when a single-ended circuit is used. Blue star $(*)$ - actual position, red triangles ( $\left.\nabla\right)$ - ideal position, (a) simulated, and b) measured.

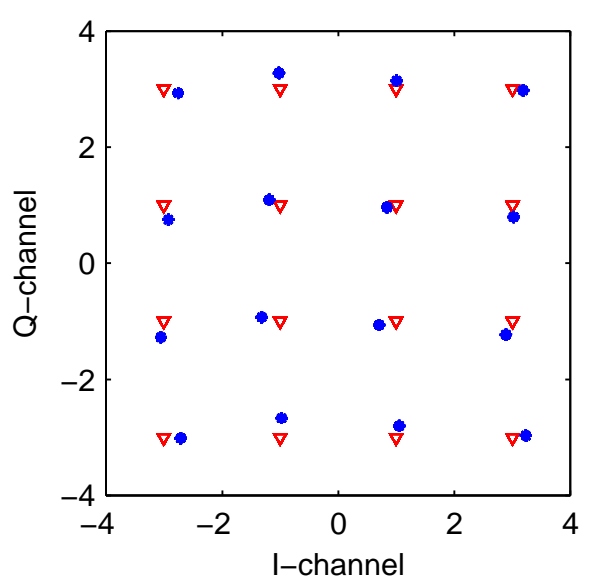

(a)

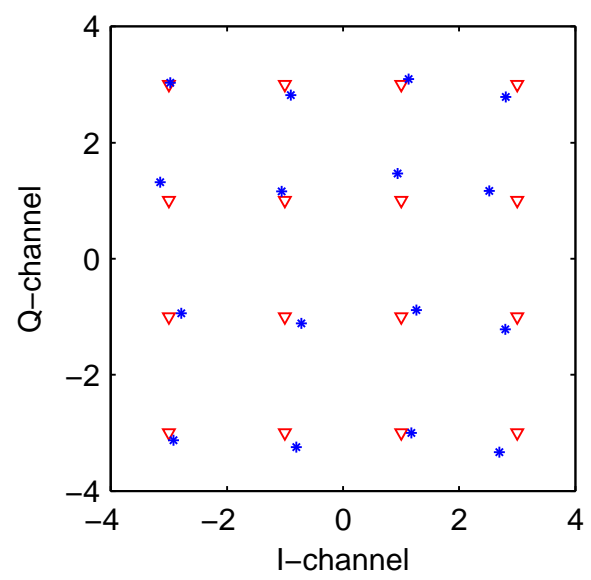

(b)

Figure 7. Constellation at $f=0.8 f_{0}$ when a differential circuit is used. Blue star $(*)$ - actual position, red triangles $(\nabla)$ - ideal position, (a) simulated, and (b) measured.

\section{CONCLUSION}

Both single-ended and differentially signaled six-port modulator architectures were investigated to give a performance comparison of state-of-the-art solutions. The single-ended and differential six-port modulators are compared, both from theory, simulation and measurements. The modulation perfor- 
mance in a single-ended six-port modulator is mainly limited due to non-ideal performance in the couplers in the six-port correlator. When a differential six-port modulator is used, the effect of non-ideal couplers reduces compared to the single-ended case and hence, allowing for better modulation performance over a wider frequency range. Carrier leakage is also suppressed when a differential six-port circuit is used due to the balanced (differential) signaling. For an EVM of less than $10 \%$, the relative bandwidth was measured to be $12 \%$ for the single-ended modulator and $30 \%$ for the differential modulator.

\section{ACKNOWLEDGMENT}

Agilent Technologies Inc. is acknowledged for their longterm technical support to Linköping University in Norrköping, Sweden. G. Knutsson at Linköping University in Norrköping, Sweden is acknowledged for his assistance and support with manufacturing of the prototypes.

\section{REFERENCES}

[1] T. Hentschel, "The six-port as a communications receiver," IEEE Transactions on Microwave Theory and Techniques, vol. 53, no. 3, pp. 10391047,2005

[2] N. K. Mallat, E. Moldovan, K. Wu, and S. O. Tatu, "Millimeterwave ultra-wideband six-port receiver using cross-polarized antennas," EURASIP J. Wirel. Commun. Netw., vol. 2009, pp. 25:1-25:7, January 2009. [Online]. Available: http://dx.doi.org/10.1155/2009/508678

[3] S. O. Tatu, E. Moldovan, K. Wu, and R. G. Bosisio, "A new direct millimeter-wave six-port receiver," IEEE Transactions on Microwave Theory and Techniques, vol. 49, no. 12, pp. 2517-2522, 2001.

[4] J. Li, R. G. Bosisio, and K. Wu, "A six-port direct digital millimeter wave receiver," in Proc. IEEE MTT-S Int. Microwave Symp. Digest, 1994, pp. 1659-1662.

[5] Y. Zhao, C. Viereck, J. F. Frigon, R. G. Bosisio, and K. Wu, "Direct quadrature phase shift keying modulator using six-port technology," Electronics Letters, vol. 41, no. 21, pp. 1180-1181, 2005.

[6] J. Osth, A. Serban, O. Owais, M. Karlsson, S. Gong, J. Haartsen, and P. Karlsson, "Diode configurations in six-port receivers with simplified interface to amplifier and filter," in Proc. IEEE Int Ultra-Wideband (ICUWB) Conf, vol. 1, 2010, pp. 1-4.

[7] J. Osth, O. Owais, M. Karlsson, A. Serban, and S. Gong, "Data and carrier interleaving in six-port receivers for increased data rate," in Proc. IEEE Int Ultra-Wideband (ICUWB) Conf, vol. 1, 2010, pp. 1-4.

[8] B. Luo and M. Y.-W. Chia, "Performance analysis of serial and parallel six-port modulators," IEEE Transactions on Microwave Theory and Techniques, vol. 56, no. 9, pp. 2062-2068, 2008.

[9] B. Luo and M. Y. W. Chia, "Direct 16 qam six-port modulator," Electronics Letters, vol. 44, no. 15, pp. 910-911, 2008.

[10] H.-S. Lim, W.-K. Kim, J.-W. Yu, H.-C. Park, W.-J. Byun, and M.-S. song, "Compact six-port transceiver for time-division duplex systems," IEEE Microwave and Wireless Components Letters, vol. 17, no. 5, pp. 394-396, 2007.

[11] A. Serban, J. Osth, Owais, M. Karlsson, S. Gong, J. Haartsen, and P. Karlsson, "Six-port transceiver for 6-9 GHz ultrawideband systems," Microwave and Optical Technology Letters, vol. 52, no. 3, pp. 740-746, 2010. [Online]. Available: http://dx.doi.org/10.1002/mop.25021

[12] J. Osth, O. Owais, M. Karlsson, A. Serban, and S. Gong, "Schottky diode as high-speed variable impedance load in six-port modulators," in Proc. IEEE Int Ultra-Wideband (ICUWB) Conf, 2011, pp. 68-71.

[13] J. Osth, Owais, M. Karlsson, A. Serban, S. Gong, and P. Karlsson, "Direct carrier six-port modulator using a technique to suppress carrier leakage," IEEE Transactions on Microwave Theory and Techniques, vol. 59, no. 3, pp. 741-747, 2011.

[14] A. Koelpin, G. Vinci, B. Laemmle, D. Kissinger, and R. Weigel, "The six-port in modern society," IEEE Microwave Magazine, vol. 11, no. 7, pp. 35-43, 2010.

[15] J. Osth, A. Serban, O. Owais, M. Karlsson, S. Gong, J. Haartsen, and P. Karlsson, "Six-port gigabit demodulator," IEEE Transactions on Microwave Theory and Techniques, vol. 59, no. 1, pp. 125-131, 2011.
[16] J. Osth, M. Karlsson, A. Serban, and S. Gong, "Carrier leakage suppression and EVM dependence on phase shifting network in six-port modulator," in Microwave and Millimeter Wave Technology (ICMMT), 2012 International Conference on, vol. 4, 2012, pp. 1-4.

[17] S. Gong, M. Karlsson, A. Serban, J. Osth, Owais, J. Haartsen, and P. Karlsson, "Radio architecture for parallel processing of extremely high speed data," in Proc. IEEE Int. Conf. Ultra-Wideband ICUWB 2009 2009, pp. 433-437.

[18] W. Ciccognani, M. Ferrari, F. Giannini, and E. Limiti, "A novel broadband mmic vector modulator for v-band applications," Int. J. RF Microw. Comput.-Aided Eng., vol. 20, pp. 103-113, January 2010. [Online]. Available: http://portal.acm.org/citation.cfm?id=1687193.1687201

[19] J. Osth, M. Karlsson, A. Serban, and S. Gong, "M-qam six-port modulator using only binary baseband data, electrical or optical," IEEE Transactions on Microwave Theory and Techniques, vol. 61, no. 6, pp. 2506-2513, June 2013.

[20] D. S. McPherson and S. Lucyszyn, "Vector modulator for w-band software radar techniques," IEEE Transactions on Microwave Theory and Techniques, vol. 49, no. 8, pp. 1451-1461, 2001.

[21] G. Brenna, D. Tschopp, J. Rogin, I. Kouchev, and Q. Huang, "A 2$\mathrm{GHz}$ carrier leakage calibrated direct-conversion wcdma transmitter in 0.13-um cmos," IEEE Journal of Solid-State Circuits, vol. 39, no. 8, pp. $1253-1262,2004$.

[22] A. Loke and F. Ali, "Direct conversion radio for digital mobile phonesdesign issues, status, and trends," IEEE Transactions on Microwave Theory and Techniques, vol. 50, no. 11, pp. 2422-2435, 2002.

[23] O. Owais, J. Osth, A. Serban, M. Karlsson, and S. Gong, "Differential six-port demodulator," Microwave and Optical Technology Letters, vol. 53, no. 9, pp. 2192-2197, 2011. [Online]. Available: http://dx.doi.org/10.1002/mop.26191

[24] O. Owais, J. Osth, and S. Gong, "Differential six-port modulator," in Proc. Int Wireless Communications and Signal Processing (WCSP) Conf, 2011, pp. 1-4.

[25] S. Y. Zheng, W. S. Chan, and K. F. Man, "Broadband phase shifter using loaded transmission line," IEEE Microwave and Wireless Components Letters, vol. 20, no. 9, pp. 498-500, 2010.

[26] Q. Gu, RF System Design of Transceivers for Wireless Communications. Secaucus, NJ, USA: Springer-Verlag New York, Inc., 2006.

[27] F. Razzaz, M. A. S. Alkanhal, and A.-F. Sheta, "UWB Wilkinson power divider using tapered transmission lines," in Proc. Progress In Electromagnetics Research Symposium. PIERS, August 2012, pp. 882884 . 


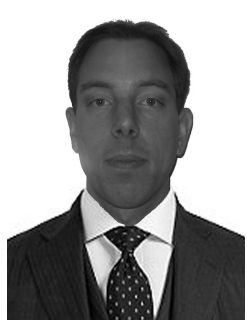

sions.

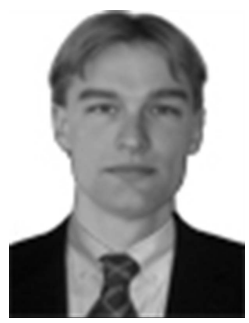

Magnus Karlsson was born in Västervik, Sweden in 1977. He received his M.Sc., Licentiate of Engineering and Ph.D. degrees from Linköping University in Sweden, in 2002, 2005 and 2008, respectively. In 2003 he started in the Communication Electronics research group at Linköping University and is currently working as a senior researcher. His main work involves wideband antenna-techniques, wideband transceiver front-ends, and wireless communications.

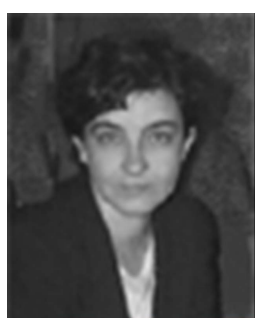

Adriana Serban received an M.Sc degree in electronic engineering from Politehnica University, Bucharest, Romania. From 1981 to 1990 she was with Microelectronica Institute, Bucharest as a Principal Engineer and was involved in mixed integrated circuit design. From 1992 to 2002 she was with Siemens AG, Munich, Germany and with Sicon AB, Linköping, Sweden as analog and mixed signal integrated circuit Senior Design Engineer. Since 2002 she has been a Lecturer at Linköping University teaching in analog/digital system design and RF circuit design. She is recived her Ph.D. degree in Communication Electronics 2010. Her main research interest has been RF circuit design and high-speed integrated circuit design.

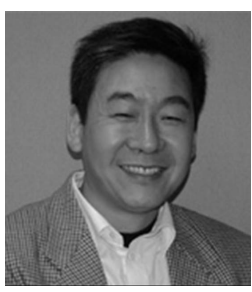

Shaofang Gong was born in Shanghai, China, in 1960. He received his B.Sc. degree from Fudan University in Shanghai in 1982, and the Licentiate of Engineering and Ph.D. degrees from Linköping University in Sweden, in 1988 and 1990, respectively. Between 1991 and 1999 he was a senior researcher at the microelectronic institute - Acreo in Sweden. From 2000 to 2001 he was the CTO at a spin-off company from the institute. Since 2002 he has been full professor in communication electronics at Linköping University, Sweden. His main research interest has been communication electronics including RF design, wireless communications and high-speed data transmissions. 